Asian Spine Journal

Vol. 5, No. 2, pp 100 106, 2011

doi:10.4184/asj.2011.5.2.100

\title{
Posterior Decompression and Fusion in Patients with Multilevel Lumbar Foraminal Stenosis: A Comparison of Segmental Decompression and Wide Decompression
}

\author{
Yoon Jae Seong ${ }^{1,2}$, Jung Sub Lee ${ }^{1,3}$, Kuen Tak Suh ${ }^{1,2}$, Jeung II Kim,3, Jong Min Lim,3, Tae Sik Goh',3 \\ ${ }^{1}$ Medical Research Institute, Pusan National University School of Medicine, Busan, Korea \\ ${ }^{2}$ Department of Orthopaedic Surgery, Pusan National University Yangsan Hospital, Yangsan, Korea \\ ${ }^{3}$ Department of Orthopaedic Surgery, Pusan National University School of Medicine, Busan, Korea
}

Study Design: This is a prospective study.

Purpose: We compared the outcomes of segmental decompression and wide decompression in patients who had multilevel lumbar foraminal stenosis with back pain.

Overview of Literature: Wide decompression and fusion in patients with multilevel lumbar foraminal stenosis may increase the risk of perioperative complications.

Methods: From March 2005 to December 2007, this study prospectively examined 87 patients with multilevel lumbar foraminal stenosis and who were treated by segmental or wide decompression along with posterior fusion using pedicle screw fixation, and these patients could be followed-up for a minimum of 2 years. Of the 87 patients, 45 and 42 patients were assigned to the segmental decompression group (group 1) and the wide decompression group (group 2), respectively. We compared the clinical and radiological outcomes of the patients in these two groups.

Results: There were no significant differences between groups 1 and 2 in terms of the levels of postoperative pain based on the visual analogue scale, the Oswestry Disability Score, the clinical results based on the Kirkaldy-Willis Criteria, the complication rate or the posterior fusion rate. On the other hand, the mean operating times in groups 1 and 2 were $153 \pm 32$ minutes and $187 \pm 36$ minutes, respectively $(p<0.05$ ). The amount of blood loss during surgery and on the first postoperative day was $840 \pm 236 \mathrm{ml}$ and $1,040 \pm 301 \mathrm{ml}$ in groups 1 and 2 , respectively $(p<0.05$ ).

Conclusions: These results suggest that segmental decompression offers promising and reproducible clinical and radiological results for patients suffering from multilevel lumbar foraminal stenosis.

Key Words: Multilevel lumbar foraminal stenosis, Segmental decompression, Wide decompression

\section{Introduction}

The goals of surgical treatment for lumbar spinal stenosis include relief of leg and back pain. Although decompres- sion is a standard treatment regimen for the surgical treatment of lumbar spinal stenosis, additional fusion after extensive decompression can be required in many cases. In particular, an extensive facetectomy is needed for decompression of the foraminal stenosis in many cases. Thus, for

Received Sep 15, 2010; 1st Revised Nov 6, 2010; Accepted Nov 10, 2010

Corresponding author: Jung Sub Lee, MD

Department of Orthopaedic Surgery, Pusan National University School of Medicine,

1-10 Ami-dong, Seo-gu, Busan 602-739, Korea

Tel: +82-51-240-7248, Fax: +82-51-247-8395, E-mail: jungsublee@ pusan.ac.kr 
cases of lumbar foraminal stenosis, a combination of neural decompression and spinal fusion can be performed to achieve the goals of surgical treatment. However, these surgeries are accompanied by substantial complications in patients with multilevel lumbar foraminal stenosis $[1,2]$.

Lumbar spinal stenosis is usually encountered in the elderly population. Elderly patients often have medical comorbidities. Old age and co-morbidities are associated with a higher complication rate [1,3,4], and these higher complication rates make it difficult for surgeons to select an appropriate surgical procedure for multilevel lumbar spinal stenosis.

Several surgical options have been performed in patients with spinal stenosis [5-12]. Although decompression surgery is essential for treating the symptoms of neurogenic claudication in patients with spinal stenosis, it is difficult to reduce back pain by decompression without fusion surgery in patients who have multilevel foraminal stenosis with back pain. However, in patients with multilevel lumbar foraminal stenosis, wide decompression and fusion may increase the risk of perioperative complications such as blood loss, infection and a prolonged operation time. Therefore, we attempted segmental decompression and fusion in patients who had multilevel lumbar foraminal stenosis with back pain and we compared the outcomes of segmental decompression and wide decompression.

\section{Materials and Methods}

From March 2005 to December 2007, we prospectively examined a total of 100 patients who had multilevel lumbar foraminal stenosis (more than 1 level) and back pain, and these patients were treated by segmental or wide decompression along with multilevel posterolateral fusion using pedicle screw fixation. Thirteen patients were lost to fol- low-up and so they were excluded. In total, 87 patients with a minimum follow-up of 2 years were enrolled in this study. The inclusion criteria were disabling back and leg pain with neurological symptoms that were refractory to at least 6 months of conservative treatment, and a moderate-to-severe degree of central and foraminal stenosis at multiple levels according to magnetic resonance imaging. The exclusion criteria were a spinal fracture, infection, tumor, revision, the possibility of secondary gains from surgical fusion or lytic spondylolisthesis. The litigious cases, i.e., traffic accidents and worker's compensation, were also excluded.

The patients were randomly assigned to 2 treatment groups using precoded sealed envelopes containing serial numbers ranging from 1 to 100 . The envelopes were not opened until the first incision. The patients with odd and even serial numbers were placed in groups 1 and 2, respectively. Of the 87 patients, 45 and 42 patients were assigned to the segmental decompression group (group 1) and the wide decompression group (group 2), respectively. All 87 patients showed back and leg pain. Associated chronic medical illness was present in 21 and 20 patients in groups 1 and 2, respectively. The patients' age, gender distribution, surgery level, smoking status, and associated medical illnesses were similar in the two groups (Table 1).

\section{Surgical techniques}

One surgeon performed all the operations using two operative techniques. The group 1 patients were treated by segmental decompression, including partial laminectomy (a cutting edge was used to remove the inferior $1 / 3$ laminae at the superior level of the decompression and the superior $1 / 3$ laminae at the inferior level of the decompression. For multilevel decompression, the mid 1/3 lamina was always preserved, total facetectomy and foraminotomy in the stenotic

Table 1. Details of the patients

\begin{tabular}{llll}
\hline \hline & Group 1 & Group 2 & $p$-value \\
\hline Age (yr) & $57.9 \pm 6.1$ & $59.7 \pm 5.2$ & 0.1601 \\
Female/Male & $35 / 10$ & $32 / 10$ & 0.9369 \\
Surgery levels & $2(22), 3(16), 4(7)$ & $2(21), 3(15), 4(6)$ & 0.9856 \\
Patients who smoke & 8 & 7 & 0.8832 \\
Medical illness & & & 0.8997 \\
$\quad$ Patients with cardiovascular & 13 & 5 & \\
$\quad$ Patients with diabetes & 6 & 1 & \\
$\quad$ Patients with other & 2 & & \\
\hline
\end{tabular}

There were no significant differences between the groups, as calculated using the $t$ - and $\boldsymbol{x}^{2}$ tests. 

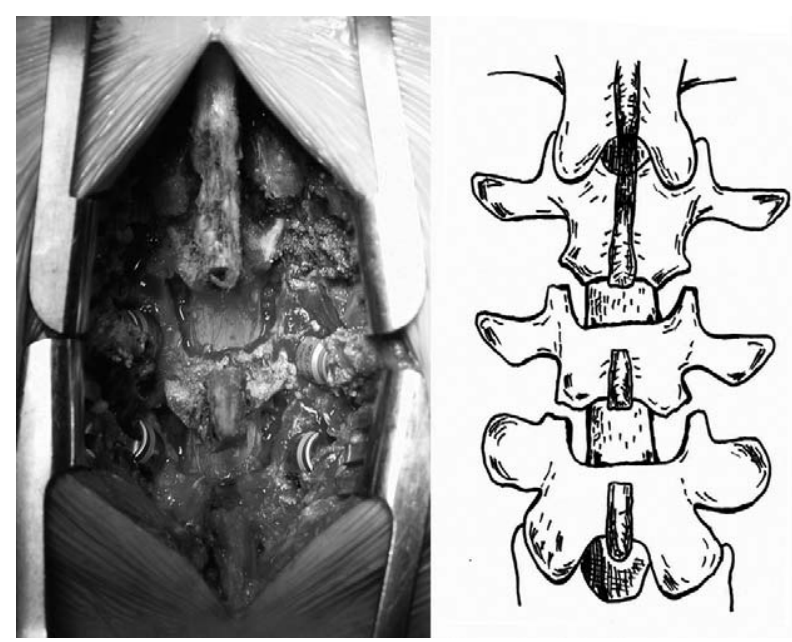

Fig. 1. The intraoperative photograph and schematic drawing show the segmental decompression in a patient with L3-5 spinal stenosis.

areas (Fig. 1). The group 2 patients were treated by wide decompression, including total laminectomy, total facetectomy and foraminotomy in the stenotic areas. All the patients underwent instrumented fusion surgery using pedicle screw/rod instrumentation. Concomitant posterolateral fusion was performed in all the patients. Allogenous cancellous bone and local bone from the lamina, spinous process and facet were used in both groups. All the patients were ambulated in a few days, with being protected by a low profile thoracic lumbar sacral orthosis (TLSO), for an early return to the activities of daily living. The TLSO was worn for 3 months after surgery.

\section{Clinical assessment}

The clinical outcomes were evaluated according to the improvement of back and leg pain, the disability and the overall clinical results. Back and leg pain was measured using a 10-point visual analog scale before surgery, postoperatively at 6 months and at the first and second postoperative year. Disability was assessed using the Oswestry Disability Questionnaire [13] before surgery and at the first and second postoperative year. The overall clinical evaluation was done based on the Kirkaldy-Willis criteria [14] at the first and second postoperative year. In addition, the operating time, the amount of blood loss during surgery and on the first postoperative day, and the incidence of complications were examined.

Complications were defined as any event requiring specific treatment. The complications were categorized as early perioperative ( $<3$ months after surgery) or late complications and as major or minor complications. Major complications were defined as conditions that adversely affected the recovery of the patient. A minor complication was defined as one that did not affect the patient's recovery. Major complications included pulmonary embolism, respiratory distress, epidural hematoma, postoperative infection, and neurologic deficits. Minor complications included urinary tract infection, ileus, and transient delirium.

The patients who exhibited symptomatic adjacent segment disease were categorized as having adjacent segment disease. The patients who demonstrated radiographic abnormalities without symptoms were excluded. Symptomatic adjacent segment disease was defined as the redevelopment of low back pain and/or radiating pain to the legs and this persisted for more than 6 weeks despite conservative treatment.

\section{Radiographic assessment}

Posterior fusion grading (A to D, with A noting solid bilateral fusion and D noting the absence of fusion) was performed using the latest anteroposterior radiograph, as described by Lenke et al. [15]. Posterior pseudarthrosis was defined as the presence of a grade $\mathrm{C}$ or $\mathrm{D}$.

\section{Statistics}

All the data was examined using the SPSS ver. 11.0 (SPSS Inc., Chicago, IL, USA). For the noncontinuous variables, a chi-square test was used to calculate the statistical significance. Repeated ANOVA tests were used to examine the statistical significance within each group. Student's ttests and chi-square tests were used to determine the statistical significance between the groups. A $p$-value $<0.05$ was considered significant.

\section{Results}

\section{Clinical results}

Both groups showed significant reductions in their low back and leg pain $(p<0.001)$. There were no significant differences between the two groups $(p>0.05)$. Both groups showed similar improvement of the Oswestry Disability Score $(p>0.05)$ (Table 2). According to the overall clinical results based on the Kirkaldy-Willis criteria, $82.2 \%$ and 
Table 2. Pain and disability scores during follow-up

\begin{tabular}{llll}
\hline \hline & Group 1 & Group 2 & $p$-value \\
\hline Pain & & & \\
$\quad$ Preoperative & $7.0 \pm 1.1$ & $7.0 \pm 1.3$ & 0.9374 \\
6 mon & $1.3 \pm 1.1$ & $1.6 \pm 1.6$ & 0.2338 \\
$1 \mathrm{yr}$ & $1.5 \pm 1.0$ & $1.7 \pm 1.4$ & 0.2811 \\
$2 \mathrm{yr}$ & $1.7 \pm 1.2$ & $1.9 \pm 1.5$ & 0.2945 \\
Oswestry & & & \\
$\quad$ Preoperative & $65 \pm 12.2$ & $67 \pm 11.9$ & 0.4315 \\
$1 \mathrm{yr}$ & $25 \pm 8.2$ & $26 \pm 11.5$ & 0.3571 \\
$2 \mathrm{yr}$ & $25 \pm 8.7$ & $27 \pm 8.4$ & 0.2727 \\
\hline
\end{tabular}

The level of pain was measured using a 10-point visual analog scale (VAS), and functional disability was assessed with the Oswestry Disability Questionnaire. The VAS score ranged from 0 to 10 (maximum pain), and the Oswestry score ranged from 0 to 100 (maximum severity). Repeated ANOVA was used to calculate the differences within each group during the follow-up. Significant differences between the preoperative and postoperative scores were found in each group $(p<0.05)$. A t-test was used to calculate the differences between the groups. No significant differences were found between the groups.

$84.4 \%$ of the group 1 patients showed good or excellent results at the first and second postoperative year, respectively. The corresponding figures in group 2 were $78.6 \%$ and $76.2 \%$, respectively (Table 3 ).

The postoperative complications are summarized in Table 4. There were 6 early and 7 late complications in group 1 , and 8 early and 8 late complications in group 2 . One superficial infection (group 1) was treated by dressing change and 1 postoperative deep infection (group 2) was treated with incision and drainage and antibiotics. The transient nerve palsies, which occurred in 1 (group 1) and 2 patients (group 2), were improved spontaneously during the followup. A surgery for hematoma evacuation was needed in 1 patient (group 2) with postoperative cauda equine syndrome. His symptoms were improved by 2 years after operation. The rate of developing early complications was not found to be related with age, the operative time, the amount of blood loss, the number of fusion levels and the number of medical comorbidities in either study group.

Adjacent segment disease developed at the proximal segments in 2 patients (one in group 1 and 1 in group 2), but they refused revision surgery. No revision surgery for adjacent segment disease was performed in either of the 2 groups.

The mean operating times were $153 \pm 32$ minutes and
Table 3. Patient overall ratings of results using Kirkaldy-Willis criteria

\begin{tabular}{ccc}
\hline \hline & $\begin{array}{c}\text { No. at 1 yr } \\
\text { postoperatively (\%) }\end{array}$ & $\begin{array}{c}\text { No. at 2 yr } \\
\text { postoperatively (\%) }\end{array}$ \\
\hline Group 1 & & \\
Successful & & \\
Excellent & $9(20)$ & $10(22)$ \\
Good & $28(62)$ & $28(62)$ \\
Unsuccessful & & \\
Fair & $8(18)$ & $6(13)$ \\
Poor & $0(0)$ & \\
Group 2 & & $6(14)$ \\
Successful & & \\
Excellent & $8(19)$ & $26(62)$ \\
Good & $25(60)$ & $9(21)$ \\
Unsuccessful & & $1(2)$ \\
Fair & $8(19)$ & 0.6655 \\
Poor & $1(2)$ & \\
$p$-value & 0.7707 & \\
\hline
\end{tabular}

There were no significant differences between the groups, as calculated using the $\boldsymbol{x}^{2}$ test.

Table 4. Complications

\begin{tabular}{lcc}
\hline \hline Complication & Group 1 & Group 2 \\
\hline Early perioperative complications & & \\
$\quad$ Pulmonary embolism & 0 & 0 \\
Ileus & 2 & 3 \\
Urinary tract infection & 1 & 0 \\
Transient delirium & 1 & 1 \\
Epidural hematoma & 0 & 1 \\
Wound infection & 1 & 1 \\
Respiratory distress syndrome & 0 & 0 \\
Neurologic deficit & 1 & 2 \\
Major complication & 2 & 4 \\
Minor complication & 4 & 4 \\
Late complications & & \\
Pseudarthrosis & 6 & 7 \\
Adjacent segment disease & 1 & 1 \\
\hline
\end{tabular}

$187 \pm 36$ minutes in groups 1 and 2 , respectively. The difference in the mean operating time was significant $(p<$ 0.05 ). The amount of blood loss during surgery and on the first postoperative day in groups 1 and 2 was $840 \pm 236 \mathrm{ml}$ and 1,040 $\pm 301 \mathrm{ml}$, respectively. The difference between the 2 groups was significant $(p<0.05)$.

The rates of complaints about a palpable spinous process in the upper instrumented spinal level were $0 \%(0 / 45)$ in group 1 and 29\% (12/42) in group 2. The difference between the 2 groups was significant $(p<0.05)$. 
Table 5. Fusion rate during follow-up assessments

\begin{tabular}{llll}
\hline \hline & Group 1 & Group 2 & $p$-value \\
\hline Posterior fusion & & & \\
No. at 1 yr postoperatively (\%) & $36(80)$ & $33(79)$ & 0.9200 \\
No. at 2 yr postoperatively (\%) & $39(87)$ & $35(83)$ & 0.8927 \\
\hline
\end{tabular}

There were no significant differences between the groups, as calculated using the $\boldsymbol{x}^{2}$ test.

\section{Radiological results}

In group 1, the posterior fusion rate was $80 \%$ and $87 \%$ in the first and second postoperative year, respectively. The corresponding figures in group 2 were $79 \%$ and $83 \%$, respectively. The posterior fusion rates of the 2 groups were similar $(p>0.05)$ (Table 5).

\section{Discussion}

Lumbar spinal stenosis is the most common indication for spinal surgery in the old age population. The aim of the treatment is the decompression of neural structures that are mechanically compressed by the degenerative tissues that form in the spinal canal and the intervertebral foramina.

Several surgical options are available to treat spinal stenosis, but wide decompressive laminectomy is most often used to decompress neural structures. To avoid destabilization of the spinal column postoperatively, alternative decompression techniques have been established to maintain the posterior bony and ligamentous elements. These techniques include selective single laminotomy, multiple unilateral or bilateral laminotomy, multilevel partial laminectomy and lumbar laminoplasty [7,8,10]. Although the goal of treatment of spinal stenosis is to decompress the affected neural structure, it is difficult to reduce back pain and neurological symptoms by decompression without performing fusion surgery in the patients who have multilevel foramianl steonosis with back pain. In many cases in whom the stenotic area is extensive or it extends to the intervertebral foramen or its lateral side, a complete decompression can not be obtained without sacrificing of the facet joint. Furthermore, bony fusion would yield a better treatment outcome for the cases with concurrent instability or potential discogenic back pain. For the cases of severe spinal stenosis involving the intervertebral foramen, minimally invasive spinal surgery cannot achieve complete decompression due to the restricted extent of the surgical vision.
In addition, the problem of bone re-growth with clinically significant recurrent stenosis is more common when restricted bone removal techniques have been employed [16]. Although the efficacy of fusion in degenerative disease remains controversial, several studies have recommended instrumentation to improve the fusion rate and the long-term clinical outcome [3,17]. Furthermore, spinal fusion may prevent recurrent stenosis. In this study, the fusion rates and clinical results of segmental decompression with posterolateral fusion were similar to those of wide decompression. No case of recurrent stenosis was encountered in either group, which suggests that segmental decompression with posterolateral fusion could be one of the treatment options for multilevel foraminal stenosis patients with back pain and that stabilization of the decompressed spine segment might prevent recurrent stenosis.

Several studies have assessed the risk of complications occurring in elderly patients after spinal surgery [18-21]. The complication rates are generally known to be correlated with age, the presence of a medical comorbidity, increased blood loss and the number of levels fused. Carreon et al. [1] concluded that elderly patients were at an increased risk of developing surgery-related complications and Deyo et al. [19] found that the morbidity and mortality rates increased with age. Conversely, some authors have reported no difference in either the outcome or the rate of complications between the elderly population and the younger population $[20,22]$. Thus, the effect of age or medical comorbidities on the complications of lumbar fusion remains unclear. In this study, age, the operative time, the amount of blood loss, the number of fusion levels and the number of medical comorbidities were found to be unrelated to the development of early complications in either of the study groups.

Wide decompression and fusion may be accompanied by substantial complications such as postoperative blood loss, a prolong operation time and postoperative infection. Further, long segment fusion and abundant blood loss may increase the incidences of complications. In this study, to reduce the rates of these complications, we performed segmental decompression of the neural structures that were mechanically compressed by the degenerative tissues. During this procedure, the mid-1/3 lamina was always preserved in the segmental decompression group because severe compression of the dural sac was not observed in this area. The early complication rates of groups 1 and 2 were similar at 13\% (6/45) and 19\% (8/42), respectively. However, the operating time and amount of blood loss were greater 
in group 2 than that in group 1. In addition, the complaint of a palpable spinous process in the upper instrumented spinal level was found to be characteristic of wide decompression. The rates of complaints about a palpable spinous process in the upper instrumented spinal level were $0 \%(0 / 45)$ in group 1 and 29\% (12/42) in group 2, which suggests that segmental decompression provides better cosmesis than wide decompression.

Some potential limitation of this study should be considered. The number of patients included was relatively small and the period of follow-up was relatively short. This study evaluated the patients' low back and leg pain together, although the patients with foraminal stenosis can have different pain intensities in the low back and leg pain. This point is one of the drawbacks in this study. Studies with a larger number of patients and a longer follow-up period should be performed in order to determine the utility of segmental decompression and fusion in patients with multilevel lumbar foraminal stenosis.

\section{Conclusions}

We found that segmental decompression, when applied with posterior instrumented fusion, offers promising and reproducible clinical/radiological results in patients who are suffering from multilevel foraminal stenosis. Moreover, despite performing segmental decompression, no bone regrowth sufficient to cause recurrent stenosis was observed, which we attribute to the stabilization of the spinal column.

\section{Acknowledgements}

This study was supported by Pusan National University Hospital Research Grant of 2011 years.

\section{REFERENCES}

1. Carreon LY, Puno RM, Dimar JR 2nd, Glassman SD, Johnson JR. Perioperative complications of posterior lumbar decompression and arthrodesis in older adults. J Bone Joint Surg Am 2003;85:2089-92.

2. Cho KJ, Suk SI, Park SR, et al. Short fusion versus long fusion for degenerative lumbar scoliosis. Eur Spine J 2008; 17:650-6.

3. Cassinelli EH, Eubanks J, Vogt M, Furey C, Yoo J, Bohlman HH. Risk factors for the development of periop- erative complications in elderly patients undergoing lumbar decompression and arthrodesis for spinal stenosis: an analysis of 166 patients. Spine (Phila Pa 1976) 2007;32: 230-5.

4. Pritchett JW, Bortel DT. Degenerative symptomatic lumbar scoliosis. Spine (Phila Pa 1976) 1993;18:700-3.

5. Brunon J, Chazal J, Chirossel JP, et al. When is spinal fusion warranted in degenerative lumbar spinal stenosis? Rev Rhum Engl Ed 1996;63:44-50.

6. Conley FK, Cady CT, Lieberson RE. Decompression of lumbar spinal stenosis and stabilization with Knodt rods in the elderly patient. Neurosurgery 1990;26:758-63.

7. Matsui H, Tsuji H, Sekido H, Hirano N, Katoh Y, Makiyama N. Results of expansive laminoplasty for lumbar spinal stenosis in active manual workers. Spine (Phila Pa 1976) 1992;17(3 Suppl):S37-40.

8. Postacchini F, Cinotti G, Perugia D, Gumina S. The surgical treatment of central lumbar stenosis: multiple laminotomy compared with total laminectomy. J Bone Joint Surg Br 1993;75:386-92.

9. Postacchini F. Surgical management of lumbar spinal stenosis. Spine (Phila Pa 1976) 1999;24:1043-7.

10. Sanderson PL, Getty CJ. Long-term results of partial undercutting facetectomy for lumbar lateral recess stenosis. Spine (Phila Pa 1976) 1996;21:1352-6.

11. Simmons ED Jr, Simmons EH. Spinal stenosis with scoliosis. Spine (Phila Pa 1976) 1992;17(6 Suppl):S117-20.

12. Kim EH, Kim HT. En bloc partial laminectomy and posterior lumbar interbody fusion in foraminal spinal stenosis. Asian Spine J 2009;3:66-72.

13. Jeon CH, Kim DJ, Kim SK, Kim DJ, Lee HM, Park HJ. Validation in the cross-cultural adaptation of the Korean version of the Oswestry Disability Index. J Korean Med Sci 2006;21: 1092-7.

14. Kirkaldy-Willis WH, Paine KW, Cauchoix J, McIvor G. Lumbar spinal stenosis. Clin Orthop Relat Res 1974;(99): 30-50.

15. Lenke LG, Bridwell KH, Bullis D, Betz RR, Baldus C, Schoenecker PL. Results of in situ fusion for isthmic spondylolisthesis. J Spinal Disord 1992;5:433-42.

16. Postacchini F, Cinotti G. Bone regrowth after surgical decompression for lumbar spinal stenosis. J Bone Joint Surg Br 1992;74:862-9.

17. Kornblum MB, Fischgrund JS, Herkowitz HN, Abraham DA, Berkower DL, Ditkoff JS. Degenerative lumbar spondylolisthesis with spinal stenosis: a prospective longterm study comparing fusion and pseudarthrosis. Spine 
(Phila Pa 1976) 2004;29:726-33.

18. Benz RJ, Ibrahim ZG, Afshar P, Garfin SR. Predicting complications in elderly patients undergoing lumbar decompression. Clin Orthop Relat Res 2001;(384):116-21.

19. Deyo RA, Cherkin DC, Loeser JD, Bigos SJ, Ciol MA. Morbidity and mortality in association with operations on the lumbar spine: the influence of age, diagnosis, and procedure. J Bone Joint Surg Am 1992;74:536-43.

20. Kilincer C, Steinmetz MP, Sohn MJ, Benzel EC, Bingaman W. Effects of age on the perioperative characteristics and short-term outcome of posterior lumbar fusion surgery. J Neurosurg Spine 2005;3:34-9.

21. Zheng F, Cammisa FP Jr, Sandhu HS, Girardi FP, Khan SN. Factors predicting hospital stay, operative time, blood loss, and transfusion in patients undergoing revision posterior lumbar spine decompression, fusion, and segmental instrumentation. Spine (Phila Pa 1976) 2002;27:818-24.

22. Marchesi DG, Aebi M. Pedicle fixation devices in the treatment of adult lumbar scoliosis. Spine (Phila Pa 1976) 1992; 17(8 Suppl):S304-9. 